Research Article

\title{
Quasi-Synchronization of Nonidentical Fractional-Order Memristive Neural Networks via Impulsive Control
}

\author{
Ruihan Chen ${ }^{1}{ }^{1}$ and Tianfeng Zhao ${ }^{2}$ \\ ${ }^{1}$ School of Mathematics, China University of Mining and Technology, Xuzhou 221116, China \\ ${ }^{2}$ College of Electrical Engineering, Nantong University, Nantong 226019, China \\ Correspondence should be addressed to Ruihan Chen; crhcumt@163.com
}

Received 10 December 2020; Revised 20 February 2021; Accepted 11 March 2021; Published 29 March 2021

Academic Editor: Shiping Wen

Copyright (C) 2021 Ruihan Chen and Tianfeng Zhao. This is an open access article distributed under the Creative Commons Attribution License, which permits unrestricted use, distribution, and reproduction in any medium, provided the original work is properly cited.

\begin{abstract}
This paper investigates the quasi-synchronization of nonidentical fractional-order memristive neural networks (FMNNs) via impulsive control. Based on a newly provided fractional-order impulsive systems comparison lemma, the average impulsive interval definition, and the Laplace transform, some quasi-synchronization conditions are obtained with fractional order $0<\alpha<1$. In addition, the error convergence rates and error boundary are also obtained. Finally, one simulation example is presented to show the validity of our results.
\end{abstract}

\section{Introduction}

Memristor was predicted as the fourth circuit element describing the relationship between magnetic flux and voltage by professor Chua [1] in 1971. This component was established successfully by HP Laboratories [2, 3] in 2008. Memristors are used instead of traditional resistive elements to simulate brain neuron synapses and build the memristor neural networks (MNNs) model [4-9] because they have memory characteristics. Now, it has been widely used in the field of information processing, associative memory, and image processing [10-12].

Fractional calculus originated in the $17^{\text {th }}$ century and is a generalization of integer-order calculus operations to arbitrary order calculus operations [13]. Scholars introduce fractional calculus into the study of MNNs and formed fractional-order memristive neural networks (FMNNs) model [14-16]. FMNNs can describe the memory properties of neurons more accurately and achieve many results in synchronization and stability [17-20]. The global Mittag-Leffler stabilization of a class of FMNNs with time delays was discussed under a state feedback control in [17]. Chen and Ding [20] via a sliding mode controller and fractional-order Lyapunov direct method studied projective synchronization of nonidentical FMNNs.
On the other hand, synchronization means several systems share a common dynamical process. However, connection weights of FMNNs changed according to the state dynamics and the synchronization of derive-response systems will be destroyed. When the parameter mismatches are small enough, we can control the synchronization error in a small region, which is called quasi-synchronization [21-24]. Recently, the impulsive control method has been widely used in the quasi-synchronization of chaotic systems. He et al. used a distributed impulsive control studying the quasi-synchronization problem of drive-response heterogeneous networks in [21] and the number of controlled nodes was also considered. Tang et al. [24] derived criteria for quasi-synchronization of nonidentical coupled Lur'e networks by delayed impulsive comparison principle, where synchronization errors for different impulsive effects with different functions were evaluated. Therefore, it is very meaningful to study the synchronization problem in the case of parameter mismatch [25-28].

To the best of our knowledge, the existing results about quasi-synchronization are concentrated on the integer-order systems, results about fractional-order systems are very few. Motivated by this, we use an impulsive controller to study the quasi-synchronization of FMNNs with parameter 
mismatches. The main innovation points of this paper are as follows: (1) a generalized fractional-order comparison lemma is provided in this paper, which plays a central role in the prove. (2) Our results connected with the fractionalorder $\alpha$, which more reflects the characteristics of FMNNs.

1.1. Notations. $\mathbb{R}$ is the space of real number, $\mathbb{N}$ is the space of nonnegative integers, $\mathscr{C}$ is the space of complex number, $\mathbb{R}^{n}$ denotes a dimensional Euclidean space, and $\mathbb{R}^{m \times n}$ is the set of $m \times n$ real matrices. For any matrix $B, B^{T}$ denotes the transpose of $\mathrm{B}$ and $I$ denotes the identity matrix. For any algebraic operations, matrices are assumed to have compatible dimensions. The notation $\|\cdot\|$ denotes the matrix 2norm or the Euclidean vector norm. Let $\mathscr{C}\left([-\infty, 0], \mathbb{R}^{n}\right)$ denotes the family of continuous functions from $[-\infty, 0]$ to $\mathbb{R}^{n} \cdot \lambda_{\text {min }}(A)$ and $\lambda_{\text {max }}(A)$ denote the minimum and maximum eigenvalue of $A$, respectively. $\operatorname{sign}(\cdot)$ is the symbolic function; $\overline{c o}\{b, c\}$ represents the closure of convex hull generated by real numbers $b$ and $c$. Define $\chi(t)$ as a continuous function except at some finite number of points $t_{k}$ at which $\chi\left(t_{k}^{+}\right)=\chi\left(t_{k}\right)$ and $\chi\left(t_{k}^{-}\right)$exist; then the set of piecewise right continuous function $\chi(t)$ is defined as $\mathrm{PC}(l)=\left\{\chi \mid \chi:[-\tau, \infty) \longrightarrow \mathbb{R}^{l}\right\}$.

\section{Preliminaries and System Description}

2.1. Caputo Fractional-Order Calculus and Mittag-Leffler Function. Caputo fractional operator plays an important role in the fractional systems and has been more practical in physical than the Riemann-Liouville fractional operation.
Therefore, we use Caputo fractional operator as the main tool in this paper.

Definition 1 (see [13]). The fractional integral of order $\alpha$ for a function $f(t)$ is defined as

$$
{ }_{0} I_{t}^{\alpha} f(t)=\frac{1}{\Gamma(\alpha)} \int_{0}^{t}(t-\tau)^{\alpha-1} f(\tau) \mathrm{d} \tau
$$

where $t \geq 0, \alpha>0$, and $\Gamma(\alpha)=\int_{0}^{+\infty} e^{-t} t^{\alpha-1} \mathrm{~d} t$.

Definition 2 (see [13]). The Caputo fractional derivative of order $\alpha$ for a function $f(t)$ is defined by

$$
{ }_{0} D_{t}^{\alpha} f(t)=\frac{1}{\Gamma(n-\alpha)} \int_{0}^{t} \frac{f^{(n)}(\tau)}{(t-\tau)^{\alpha-n+1}} \mathrm{~d} \tau,
$$

where $n-1<\alpha<n ; n$ is a positive integer. The Laplace transform of the Caputo fractional-order derivative is

$$
\mathscr{L}\left({ }_{0} D_{t}^{\alpha} f(t)\right)=s^{\alpha} \mathscr{L}(f(t))-\sum_{k=0}^{n-1} s^{\alpha-k-1} f^{(k)}(0),
$$

where $\alpha>0, n=[\alpha]+1$.

Lemma 1. Let $Z_{1}(s)$ and $Z_{2}(s) \in \mathbb{P C}(1)$ be jumping discontinuity at $s=s_{k}, s \geq 0, Z_{1}\left(s_{k}^{+}\right)$, and $Z_{2}\left(s_{k}^{+}\right)$exist, $Z_{1}\left(s_{k}\right)=Z_{1}\left(s_{k}^{-}\right), \quad Z_{2}\left(s_{k}\right)=Z_{2}\left(s_{k}^{-}\right)$. The initial value $Z_{1}(s)=Z_{2}(s)=h(0) \geq 0$. If there are constants $c_{1}, c_{2}>0$, $0<\alpha<1$, and $\mu>0$, such that

$$
\begin{aligned}
& \left\{{ }_{0} D_{t}^{\alpha} 0 Z_{1}(s) \leq c_{1} Z_{1}(s)+c_{2}, \quad s \neq s_{k}, Z_{1}\left(s_{k}^{+}\right) \leq \mu Z_{1}\left(s_{k}^{-}\right), \quad k \in \mathbb{N},\right. \\
& \left\{{ }_{0} D_{t}^{\alpha} Z_{2}(s) \geq c_{1} Z_{2}(s)+c_{2}, \quad s \neq s_{k}, Z_{2}\left(s_{k}^{+}\right)=\mu Z_{2}\left(s_{k}^{-}\right), \quad k \in \mathbb{N},\right.
\end{aligned}
$$

and $Z_{1}(s) \leq Z_{2}(s)$ for $s \leq 0$, then $Z_{1}(s) \leq Z_{2}(s)$ for $s \geq 0$.

Proof. From (4) and (5), we have

$$
\begin{aligned}
& Z_{1}(s) \leq h(0) E_{\alpha}\left(c_{1} s^{\alpha}\right)+\int_{0}^{s}(s-t)^{\alpha-1} \times E_{\alpha, \alpha}\left(c_{1}(s-t)^{\alpha}\right) c_{2} \mathrm{~d} t \\
& Z_{2}(s) \geq h(0) E_{\alpha}\left(c_{1} s^{\alpha}\right)+\int_{0}^{s}(s-t)^{\alpha-1} \times E_{\alpha, \alpha}\left(c_{1}(s-t)^{\alpha}\right) c_{2} \mathrm{~d} t .
\end{aligned}
$$

We first prove

$$
Z_{1}(s) \leq Z_{2}(s), \quad\left[0, t_{1}\right)
$$

As $Z_{1}(s), Z_{2}(s)$ are continuous functions and $Z_{1}(s) \leq Z_{2}(s)$ for $s \leq 0$, according to (6) and (7), we have $Z_{2}(s) \geq Z_{1}(s)$, and therefore (8) holds. Then, assume $Z_{1}(s) \leq Z_{2}(s)$ for $t \in\left[t_{k-1}, t_{k}\right), k \leq c$, and $c \in \mathbb{N}$. Then, we have $Z_{1}\left(s_{c}\right) \leq \mu Z_{1}\left(s_{c}^{-}\right) \leq \mu Z_{2}\left(s_{c}^{-}\right)=Z_{2}\left(s_{c}\right)$. In a similar manner to the proof of (8), we can have $Z_{1}(s) \leq Z_{2}(s)$ for $s \in\left[t_{c}, t_{c+1}\right)$. By mathematical induction, it is easy to conclude that $Z_{1}(s) \leq Z_{2}(s)$ for $s \geq 0$. This completes the proof.
Lemma 2 (see [28]). $x(t) \in \mathbb{R}^{n}$ is a derivable and continuous function, for $t \geq t_{0}$; we have

$$
{ }_{t_{0}} D_{t}^{\alpha}\left[x^{T}(t) M x(t)\right] \leq 2 x^{T}(t) M_{t_{0}} D_{t}^{\alpha} x(t), \quad \alpha \in(0,1]
$$

where $M \in \mathbb{R}^{n \times n}$ is a positive definite matrix.

The Mittag-Leffler function is defined in the following.

Definition 3 (see [13]). Two-parameter Mittag-Leffler function is defined as 


$$
E_{\alpha, \beta}(t)=\sum_{k=0}^{\infty} \frac{t^{k}}{\Gamma(k \alpha+\beta)},
$$

where $\alpha>0, \beta>0$, and $t \in \mathscr{C}$. When $\beta=0$, we have

$$
E_{\alpha}(t)=\sum_{k=0}^{\infty} \frac{t^{k}}{\Gamma(k \alpha+1)}
$$

where $\alpha>0, t \in \mathscr{C}$.

Lemma 3 (see [29]). $t \in \mathbb{R}, t>0$, and $0<\alpha<1 . E_{\alpha}(t)$ is a monotone increasing function. For any integer $N>1$, and $t \neq 0,|\arg (t)|>(\pi / 2)$, then we have the following asymptotic expansion:

$$
E_{\alpha, \beta}(t) \leq \frac{1}{\alpha} t^{(1-\beta) / \alpha} e^{t^{(1 / \alpha)}}-\sum_{p=1}^{N} \frac{t^{-p}}{\Gamma(\beta-\alpha p)}+O\left(\frac{1}{t^{N+1}}\right) .
$$

So, there exists $t_{0}>0$, when $t>t_{0}$, and we have

$$
\begin{aligned}
E_{\alpha}(t) & \leq \frac{1}{\alpha} e^{t^{(1 / \alpha)}}, \\
E_{\alpha, \alpha}(t) & \leq \frac{1}{\alpha} t^{(1-\alpha) / \alpha} e^{t^{(1 / \alpha)}} .
\end{aligned}
$$

2.2. System Description. Now, we consider the following FMNNs as a drive system:

$$
{ }_{0} D_{t}^{\alpha} x_{i}(t)=-d_{i} x_{i}(t)+\sum_{j=1}^{n} b_{i j}\left(x_{j}(t)\right) f_{j}\left(x_{j}(t)\right) x_{i}(0)=x_{0 i},
$$

where $i, j \in \mathbb{N} ; \alpha$ is the fractional order; $x_{i}(t)$ represents the state variable of the $i$ th neuron; $d_{i}>0$ denotes the selffeedback connection weight; $x_{0}=\left(x_{01}, x_{02}, \ldots, x_{0 n}\right)^{T}$ is the initial value of state vector; $f_{j}(\cdot)$ denote the activation functions; and $b_{i j}\left(x_{j}(t)\right)$ are memristive connection weights satisfy the following conditions:

$$
b_{i j}\left(x_{j}(t)\right)= \begin{cases}b_{i j}^{*}, & \left|x_{j}(t)\right| \leq \neg_{x j} \\ b_{i j}^{* *}, & \left|x_{j}(t)\right|>\neg_{x j}\end{cases}
$$

where the switching jumps $\neg_{x j}>0, b_{i j}^{*}$, and $b_{i j}^{*^{*}}$ are all constants.

The corresponding response system is described as

$$
\left\{{ }_{0} D_{t}^{\alpha} y_{i}(t)=-d_{i} y_{i}(t)+\sum_{j=1}^{n} \widetilde{b}_{i j}\left(y_{j}(t)\right) f_{j}\left(y_{j}(t)\right), y_{i}(0)=y_{0 i}\right. \text {, }
$$

where $\theta_{i}(t)$ is the impulsive controller, and the memristorbased connection weights $\widetilde{b}_{i j}\left(y_{j}(t)\right)$ satisfy the following condition:

$$
\tilde{b}_{i j}\left(y_{j}(t)\right)= \begin{cases}\widetilde{b}_{i j}^{*}, & \left|y_{j}(t)\right| \leq \tau_{y j}, \\ \tilde{b}_{i j}^{* *}, & \left|y_{j}(t)\right|>\neg_{y j},\end{cases}
$$

where $i, j \in \mathbb{N}$ and switching jumps $\neg_{y j}>0, \widetilde{b}_{i j}^{*}, \widetilde{b}_{i j}^{* *}$ are known constants.

For system (14), by applying the theories of set-valued maps and differential inclusions, we have the following differential inclusion form:

$$
{ }_{0} D_{t}^{\alpha} x_{i}(t) \in-d_{i} x_{i}(t)+\sum_{j=1}^{n} K\left[b_{i j}\left(x_{j}(t)\right)\right] f_{j}\left(x_{j}(t)\right) \text {. }
$$

Let $\widehat{b}_{i j}=\min \left\{b_{i j}^{*}, b_{i j}^{*^{*}}\right\}, \check{b}_{i j}=\max \left\{b_{i j}^{*}, b_{i j}^{*^{*}}\right\}$, and $\operatorname{co}\left\{b_{i j}^{*}\right.$, $\left.b_{i j}^{* *}\right\}=\left[\widehat{b}_{i j}, \check{b}_{i j}\right]$. The set-valued maps are defined as

$$
K\left[b_{i j}\left(x_{j}(t)\right)\right]= \begin{cases}b_{i j}^{*}, & \left|x_{j}(t)\right|<\neg_{x j}, \\ c o\left\{b_{i j}^{*}, b_{i j}^{* *}\right\}, & \left|x_{j}(t)\right|=\neg_{x j}, \\ b_{i j}^{* *}, & \left|x_{j}(t)\right|>\top_{x j},\end{cases}
$$

and, besides, there exist measurable functions $\eta_{i j}\left(x_{j}(t)\right) \in K\left[b_{i j}\left(x_{j}(t)\right)\right]$ such that

$$
{ }_{0} D_{t}^{\alpha} x_{i}(t)=-d_{i} x_{i}(t)+\sum_{j=1}^{n} \eta_{i j}\left(x_{j}(t)\right) f_{j}\left(x_{j}(t)\right) .
$$

Similarly, there exist measurable functions $\widetilde{\eta_{i j}}\left(y_{j}(t)\right)=$ $K\left[\widetilde{b}_{i j}\left(y_{j}(t)\right)\right]$ such that

$$
{ }_{0} D_{t}^{\alpha} y_{i}(t)=-d_{i} y_{i}(t)+\sum_{j=1}^{n} \widetilde{\eta_{i j}}\left(y_{j}(t)\right) f_{j}\left(y_{j}(t)\right)+\theta_{i}(t) .
$$

To ensure the uniqueness and existence of the solution of system (20) and (21), we make the following assumption.

Assumption 1. There exists a positive constant $L_{j}$ such that

$$
\left|f_{j}(x)-f_{j}(y)\right| \leq L_{j}|x-y|
$$

for all $x, y \in \mathbb{R}, j \in \mathbb{N}$.

Lemma 4 (see [30]). Let Assumption 1 be satisfied; then the following inequalities hold:

$$
\begin{aligned}
& \left|\widetilde{\eta}_{i j}\left(y_{j}(t)\right) f_{j}\left(y_{j}(t)\right)-\eta_{i j}\left(x_{j}(t)\right) f_{j}\left(x_{j}(t)\right)\right| \\
& \left.\leq \bar{b}_{i j} L_{j}\left|e_{j}(t)\right|+\left|\Delta b_{i j}\right| L_{j}\right\rceil_{\text {max }},
\end{aligned}
$$

where $\bar{b}_{i j}=\max \left\{\left|b_{i j}^{*}\right|,\left|b_{i j}^{*^{*}}\right|\right\}, \neg_{\max }=\max \left\{\neg_{x j}, \neg_{y j}\right\}, \Delta b_{i j}$ $=b_{i j}^{*}-b_{i j}^{*}$.

Remark 1. To reduce the conservativeness of our results, the constraints on activation functions $f_{j}\left( \pm \neg_{x j}\right)=f_{j}$ $\left( \pm \neg_{y j}\right)=0, g_{j}\left( \pm \neg_{x j}\right)=g_{j}\left( \pm \neg_{y j}\right)=0$ are removed in this paper.

Denote $e(t)=y(t)-x(t)$ as the synchronization error and the impulsive controller is designed as

$$
\theta_{i}(t)=-a_{i} e_{i}(t)+\sum_{k=1}^{\infty}\left(\mu_{k}-1\right) e_{i}(t) \delta\left(t-t_{k}\right),
$$


where $a_{i}$ are nonnegative constants, $i \in \mathbb{N}$; impulsive intensity $\mu_{k} \neq 0 ; \delta(\cdot)$ denotes the Dirac impulsive function; and the time series $\zeta=\left\{t_{1}, t_{2}, \ldots\right\}$ is a strictly increasing sequence of impulsive instants which satisfies $t_{k-1}<t_{k}$ and $\lim _{k \longrightarrow+\infty} t_{k}=+\infty$.
With considering the impulsive controller (24), the controlled error neural networks could be rewritten as follows:

$$
\left\{{ }_{0} D_{t}^{\alpha} e_{i}(t)=-\left(d_{i}+a_{i}\right) e_{i}(t)+\sum_{j=1}^{n}\left[\widetilde{\eta_{i j}}\left(y_{j}(t)\right) f_{j}\left(y_{j}(t)\right)-\eta_{i j}\left(x_{j}(t)\right) f_{j}\left(x_{j}(t)\right)\right], \quad t \neq t_{k}, \Delta e_{i}\left(t_{k}\right)=e_{i}\left(t_{k}^{+}\right)=\mu_{k} e_{i}\left(t_{k}^{-}\right), \quad k \in \mathbb{N},\right.
$$

where $e_{i}(t)$ is right-hand continuous at $t=t_{k}$ and the initial conditions $e_{i}(0)=e_{0 i} \in \mathscr{C}\left([-\infty, 0], \mathbb{R}^{n}\right)$ for $i \in \mathbb{N}$.

2.3. The Definition of Quasi-Synchronization and Average Impulsive Interval. Now, the definitions of quasi-synchronization and average impulsive interval are given as follows.

Definition 4 (see [24]). If there exists a compact set $\Theta$ for any $e_{i}(0) \in \mathscr{C}\left([-\infty, 0], \mathbb{R}^{n}\right)$, with the error $e(t)$ converging to $\Theta=\{\|e(t)\| \leq \varrho\}$ as $t$ goes to infinity, then system (14) will achieve quasi-synchronization with error bound $\varrho>0$.

Definition 5 (see [26]). Consider the impulsive sequence $\zeta=$ $\left\{t_{1}, t_{2}, \ldots\right\}$ in the time interval $(t, T) . \omega(t, T)$ is represented as the number of impulsive times, if there exist positive numbers $N_{0}$ and $T_{a}$, such that

$$
\frac{T-t}{T_{a}}-N_{0} \leq \omega(t, T) \leq \frac{T-t}{T_{a}}+N_{0}, \quad \forall T \geq t \geq 0,
$$

and then, $T_{a}$ is greater than the average impulsive interval of the impulsive sequence $\zeta$.

\section{Main Results}

In this part, we explore the quasi-synchronization of system (25) by impulsive controller (24). For two different impulsive intensities $-\alpha<\mu_{k} \leq \alpha$ and $\mu_{k} \neq 0$ and $\mu_{k} \leq-\alpha$ or $\mu_{k}>\alpha$, we have Theorems 1 and 2 .
Theorem 1. When impulsive intensity $-\alpha<\mu_{k} \leq \alpha$ and $\mu_{k} \neq 0$, if there exist diagonal matrices $Q>0, P>0$, scalars $\beta>0, \quad$ and $, \quad d=\operatorname{diag}\left(d_{1}, d_{2}, \ldots, d_{n}\right), \quad \bar{B}=\left(\overline{b_{i j}}\right)_{n \times n}$, $L=\operatorname{diag}\left(L_{1}, L_{2}, \ldots, L_{n}\right), \quad \pi=N^{T} P N, \quad N=\Delta B L \sim_{\max }$, $|\Delta B|=\left(\left|\Delta b_{i j}\right|\right)_{n \times n}, \quad \widetilde{\neg}_{\max }=\operatorname{diag}\left(\neg_{\max }, \neg_{\max }, \ldots, \neg_{\max }\right), \quad \iota=$ $\max _{k \in \mathbb{N}}\left\{\mu_{k}^{2}\right\}$, if

$$
\begin{aligned}
& \text { (1) }-2 Q d-2 Q a+Q \bar{B} L^{\prime}+\bar{L}^{T} \bar{B}^{T} Q+P^{-1} Q^{2}-\beta Q<0 \\
& \text { (2) } \Upsilon=\frac{\ln (1 / \alpha)}{T_{a}}+\beta^{(1 / \alpha)}<0
\end{aligned}
$$

are satisfied, then error systems (25) achieve quasi-synchronization with error bound $\sqrt{\left(\left(\left(\beta^{(1 / \alpha)-1} / \alpha\right)(\iota / \alpha)^{-N_{0}} \pi\right) /\left(\lambda_{\min }(Q) \cdot(-Y)\right)\right)}$ at the convergence rate $(h / 2)$, where $h$ is the unique solution of the equation $h+\left(\ln (\iota / \alpha) / T_{a}\right)+\beta^{(1 / \alpha)}=0$.

Proof. Construct the following Lyapunov function:

$$
V(t)=\sum_{i=1}^{n} Q_{i}\left|e_{i}(t)\right|^{2}
$$

For $t \in\left[t_{k-1}, t_{k}\right)(k=1,2, \ldots)$, according to Lemmas 2 and 4 , we get 


$$
\begin{aligned}
& { }_{0} D_{t}^{\alpha} V(t) \leq 2 \sum_{i=1}^{n}\left|e_{i}(t)\right| Q_{i} \operatorname{sign}\left(e_{i}(t)\right)_{0} D_{t}^{\alpha} V e_{i}(t) \\
& =2 \sum_{i=1}^{n}\left|e_{i}(t)\right| Q_{i} \operatorname{sign}\left(e _ { i } ( t ) \left\{-\left(d_{i}+a_{i}\right) e_{i}(t)\right.\right. \\
& +\sum_{j=1}^{n}\left[\widetilde{\eta_{i j}}\left(y_{j}(t)\right) f_{j}\left(y_{j}(t)\right)\right. \\
& \left.\left.-\eta_{i j}\left(x_{j}(t)\right) f_{j}\left(x_{j}(t)\right)\right]\right\} \\
& \leq-2 \sum_{i=1}^{n}\left|e_{i}(t)\right| Q_{i}\left(d_{i}+a_{i}\right)\left|e_{i}(t)\right| \\
& +2 \sum_{i=1}^{n} \sum_{j=1}^{n}\left|e_{i}(t)\right| Q_{i}\left|\widetilde{\eta_{i j}}\left(y_{j}(t)\right) f_{j}\left(y_{j}(t)\right)-\eta_{i j}\left(x_{j}(t)\right) f_{j}\left(x_{j}(t)\right)\right| \\
& \left.\leq-2 \sum_{i=1}^{n}\left|e_{i}(t)\right| Q_{i}\left(d_{i}+a_{i}\right)\left|e_{i}(t)\right|+2 \sum_{i=1}^{n} \sum_{j=1}^{n}\left|e_{i}(t)\right| Q_{i}\left(\bar{b}_{i j} L_{j}\left|e_{j}(t)\right|+\left|\Delta b_{i j}\right| L_{j}\right\urcorner_{\max }\right) \\
& =-2|e(t)|^{T} \mathrm{Q}(d+a)|e(t)|+2|e(t)|^{T} \mathrm{Q} \times \bar{B} L|e(t)|+2|e(t)|^{T} \mathrm{QN} \\
& \leq-2|e(t)|^{T} Q(d+a)|e(t)|+2|e(t)|^{T} Q \times \bar{B} L|e(t)|+|e(t)|^{T} P^{-1} Q^{2}|e(t)|+N^{T} P N \\
& =|e(t)|^{T}\left(-2 Q d-2 Q a+Q \bar{B} \dot{L}+\bar{L}^{T} Q+P^{-1} Q^{2}-\beta Q\right)|e(t)|+\beta|e(t)|^{T} Q|e(t)|+N^{T} P N \text {. } \\
& V\left(t_{k}^{+}\right)=\sum_{i=1}^{N} e_{i}^{T}\left(t_{k}^{+}\right) Q_{i} e_{i}\left(t_{k}^{+}\right) \\
& =\mu_{k}^{2} V\left(t_{k}^{-}\right) \text {. } \\
& { }_{0} D_{t}^{\alpha} V(t) \leq \beta \sum_{i=1}^{n} Q_{i}\left|e_{i}(t)\right|^{2}+\pi=\beta V(t)+\pi \text {. }
\end{aligned}
$$

For $t=t_{k}, k \in N$, according to (25), it yields
Let $\Lambda(t)$ be the unique solution of the following delayed impulsive comparison system for any $\gamma>0$ :

$$
\left\{{ }_{0} D_{t}^{\alpha} \Lambda(t)=\beta \Lambda(t)+\pi+\gamma, \Lambda\left(t_{k}^{+}\right)=\mu_{k}^{2} \Lambda\left(t_{k}^{-}\right), \quad k=1,2, \ldots, \Lambda(t)=\lambda_{\max }(Q)\|e(0)\|^{2}\right.
$$

When $t>0$, we have $v(t) \leq \Lambda(t)$ according to Lemma 1 . For $t \in\left[0, t_{1}\right)$, using the Laplace transform on (32), one gets

$$
\begin{aligned}
\Lambda(t)= & E_{\alpha}\left(\beta t^{\alpha}\right) \Lambda(0)+\int_{0}^{t}(t-s)^{\alpha-1} E_{\alpha, \alpha}\left(\beta(t-s)^{\alpha}\right) \\
& \times(\pi+\gamma) \mathrm{d} s .
\end{aligned}
$$

By using Lemma 3, we have

$$
\begin{gathered}
E_{\alpha}\left(\beta t^{\alpha}\right) \leq \frac{1}{\alpha} e^{\beta^{(1 / \alpha)} t}, \\
E_{\alpha, \alpha}\left(\beta(t-s)^{\alpha}\right) \leq \frac{\beta^{(1 / \alpha)-1}}{\alpha}(t-s)^{1-\alpha} e^{\beta^{(1 / \alpha)}(t-s)} .
\end{gathered}
$$

Then, we can transform (33) as

$$
\Lambda(t) \leq \frac{1}{\alpha} e^{\beta^{(1 / \alpha)} t} \Lambda(0)+\int_{0}^{t} \frac{\beta^{(1 / \alpha)-1}}{\alpha} e^{\beta^{(1 / \alpha)}(t-s)} \times(\pi+\gamma) \mathrm{d} s .
$$

For $t \in\left[t_{1}, t_{2}\right)$, one obtains from (35) and the second equation of (32) that

$$
\begin{aligned}
\Lambda\left(t_{1}^{+}\right) & \leq \frac{\iota}{\alpha} e^{\beta^{(1 / \alpha)} t_{1}} \Lambda(0)+\int_{0}^{t_{1}} \frac{\iota \beta^{(1 / \alpha)-1}}{\alpha} e^{\beta^{(1 / \alpha)}\left(t_{1}-s\right)} \times(\pi+\gamma) \mathrm{d} s \\
\Lambda(t) & \leq \frac{1}{\alpha} e^{\beta^{(1 / \alpha)}\left(t-t_{1}\right)} \Lambda\left(t_{1}^{+}\right)+\int_{t_{1}}^{t} \frac{\beta^{(1 / \alpha)-1}}{\alpha} e^{\beta^{(1 / \alpha)}(t-s)} \times(\pi+\gamma) \mathrm{d} s \\
& =\frac{\iota}{\alpha^{2}} e^{\beta^{(1 / \alpha)} t} \Lambda(0)+\frac{\iota}{\alpha^{2}} \int_{0}^{t_{1}} \beta^{(1 / \alpha)-1} e^{\beta^{(1 / \alpha)}(t-s)} \times(\pi+\gamma) \mathrm{d} s+\frac{1}{\alpha} \int_{t_{1}}^{t} \beta^{(1 / \alpha)-1} e^{\beta^{(1 / \alpha)}(t-s)} \times(\pi+\gamma) \mathrm{d} s .
\end{aligned}
$$


For $t \in\left[t_{2}, t_{3}\right)$,

$$
\begin{aligned}
\Lambda\left(t_{2}^{+}\right) \leq & \frac{l^{2}}{\alpha^{2}} e^{\beta^{(1 / \alpha)} t_{2}} \Lambda(0)+\frac{l^{2}}{\alpha^{2}} \int_{0}^{t_{1}} \beta^{(1 / \alpha)-1} e^{\beta^{(1 / \alpha)}\left(t_{2}-s\right)} \times(\pi+\gamma) \mathrm{d} s+\frac{l}{\alpha} \int_{t_{1}}^{t_{2}} \beta^{(1 / \alpha)-1} e^{\beta^{(1 / \alpha)}\left(t_{2}-s\right)} \times(\pi+\gamma) \mathrm{d} s \\
\Lambda(t) \leq & \frac{1}{\alpha} e^{\beta^{(1 / \alpha)}\left(t-t_{2}\right)} \Lambda\left(t_{2}^{+}\right)+\int_{t_{2}}^{t} \frac{\beta^{(1 / \alpha)-1}}{\alpha} e^{\beta^{(1 / \alpha)}(t-s)} \times(\pi+\gamma) \mathrm{d} s \\
= & \frac{l^{2}}{\alpha^{3}} e^{\beta^{(1 / \alpha)} t} \Lambda(0)+\frac{l^{2}}{\alpha^{3}} \int_{0}^{t_{1}} \beta^{(1 / \alpha)-1} e^{\beta^{(1 / \alpha)}(t-s)} \times(\pi+\gamma) \mathrm{d} s \\
& +\frac{l}{\alpha^{2}} \int_{t_{1}}^{t_{2}} \beta^{(1 / \alpha)-1} e^{\beta^{(1 / \alpha)}(t-s)} \times(\pi+\gamma) \mathrm{d} s \\
& +\frac{1}{\alpha} \int_{t_{2}}^{t} \beta^{(1 / \alpha)-1} e^{\beta^{(1 / \alpha)}(t-s)} \times(\pi+\gamma) \mathrm{d} s .
\end{aligned}
$$

By induction, we can derive that, for $t \in\left[t_{k}, t_{k+1}\right)$,

$$
\begin{aligned}
\Lambda(t) \leq & \prod_{0 \leq t_{k} \leq t} \frac{l}{\alpha} e^{\beta^{(1 / \alpha)}} \frac{1}{\alpha} \Lambda(0)+\int_{0}^{t} \prod_{s \leq t_{k} \leq t} \frac{l}{\alpha} e^{\beta^{(1 / \alpha)}(t-s)} \\
& \times \frac{\beta^{(1 / \alpha)-1}}{\alpha}(\pi+\gamma) \mathrm{d} s .
\end{aligned}
$$

$$
\begin{aligned}
e^{\beta^{(1 / \alpha)}(t-s)} \prod_{s \leq t_{k} \leq t} \frac{l}{\alpha} & \leq e^{\beta^{(1 / \alpha)}(t-s)}\left(\frac{l}{\alpha}\right)^{\left(\left(t-s / T_{a}\right)-N_{0}\right)} \\
& \leq\left(\frac{l}{\alpha}\right)^{-N_{0}} e^{\left(\left(\ln (1 / \alpha) / T_{a}\right)+\beta^{(1 / \alpha)}\right)(t-s)}
\end{aligned}
$$

Substituting (39) into (38) yields

According to Definition 5, we have

$$
\begin{aligned}
\Lambda(t) \leq & \left(\frac{l}{\alpha}\right)^{-N_{0}} \lambda_{\max }(Q) \sum_{i=1}^{N}\left\|e_{i}(0)\right\|^{2} \frac{1}{\alpha} e^{\left(\left(\ln (\iota / \alpha) / T_{a}\right)+\beta^{(1 / \alpha)}\right) t} \\
& +\int_{0}^{t}\left(\frac{l}{\alpha}\right)^{-N_{0}} e^{\left(\left(\ln (\iota / \alpha) / T_{a}\right)+\beta^{(1 / \alpha)}\right)(t-s)} \frac{\beta^{(1 / \alpha)-1}}{\alpha} \times(\pi+\gamma) \mathrm{d} s \\
\leq & \Omega e^{\left(\left(\ln (\iota / \alpha) / T_{a}\right)+\beta^{(1 / \alpha)}\right) t}+\int_{0}^{t} \frac{\beta^{(1 / \alpha)-1}}{\alpha} e^{\left(\left(\ln (\iota / \alpha) / T_{a}\right)+\beta^{(1 / \alpha)}\right)(t-s)} \times\left[\left(\frac{l}{\alpha}\right)^{-N_{0}}(\pi+\gamma)\right] \mathrm{d} s,
\end{aligned}
$$

where $\Omega=(\iota / \alpha)^{-N_{0}}(1 / \alpha) \lambda_{\max }(Q) \sup \|e(0)\|^{2}>0$. Define $\Delta(h)=h+\left(\left(\ln (\iota / \alpha) / T_{a}\right)+\beta^{(1 / \alpha)}\right)$. Note that $\Delta(h)$ is a continuous function, $\Delta(0)=\left(\left(\ln (\iota / \alpha) / T_{a}\right)+\beta^{(1 / \alpha)}\right)<0$, and $\dot{\Delta}(h)=1>0$. Above all, $\Delta(h)=0$ has a unique solution for $h>0$. Since $\gamma>0, h>0,0<\iota<1$, we obtain

$$
\begin{aligned}
\Lambda(t) \leq & \left(\frac{l}{\alpha}\right)^{-N_{0}} \lambda_{\max }(Q)\|e(0)\|^{2} \\
< & \Omega e^{-h t}+\left(\left(\left(\beta^{(1 / \alpha)-1} / \alpha\right)(\iota / \alpha)^{-N_{0}}(\pi+\gamma)\right) /\right. \\
& \left.\left(-\left(\left(\ln (\iota / \alpha) / T_{a}\right)+\beta^{(1 / \alpha)}\right)\right)\right),
\end{aligned}
$$

for $t \leq 0$, which implies that (41) holds for all $t>0$. Assume that if (41) does not hold for all $t>0$, then there at least exists a time instant $t^{*}>0$,

$$
\Lambda\left(t^{*}\right) \geq \Omega e^{-h t^{*}}+\frac{\left(\beta^{(1 / \alpha)-1} / \alpha\right)(\iota / \alpha)^{-N_{0}}(\pi+\gamma)}{-\left(\left(\ln (\iota / \alpha) / T_{a}\right)+\beta^{(1 / \alpha)}\right)},
$$

but, for $t<t^{*}$, we have

$$
\Lambda(t)<\Omega e^{-h t}+\frac{\left(\beta^{(1 / \alpha)-1} / \alpha\right)(\iota / \alpha)^{-N_{0}}(\pi+\gamma)}{-\left(\left(\ln (\iota / \alpha) / T_{a}\right)+\beta^{(1 / \alpha)}\right)} .
$$


Denote $\left(\ln (\iota / \alpha) / T_{a}\right)+\beta^{(1 / \alpha)}=-r$; combining (40)and (43), we have

$$
\begin{aligned}
\Lambda\left(t^{*}\right) & \leq \Omega e^{\left(\left(\ln (\iota / \alpha) / T_{a}\right)+\beta^{(1 / \alpha)}\right) t^{*}}+\int_{0}^{t^{*}} \frac{\beta^{(1 / \alpha)-1}}{\alpha} e^{\left(\left(\ln (\iota / \alpha) / T_{a}\right)+\beta^{(1 / \alpha)}\right)\left(t^{*}-s\right)} \times\left(\frac{l}{\alpha}\right)^{-N_{0}}(\pi+\gamma) \mathrm{d} s \\
& <e^{-r t^{*}}\left\{\Omega+\frac{\left(\beta^{(1 / \alpha)-1} / \alpha\right)(\iota / \alpha)^{-N_{0}}(\pi+\gamma)}{r}+\int_{0}^{t^{*}} \frac{\beta^{(1 / \alpha)-1}}{\alpha} \times e^{r s}\left(\frac{l}{\alpha}\right)^{-N_{0}}(\pi+\gamma) \mathrm{d} s\right\} \\
& \leq e^{-r t^{*}}\left\{\Omega+\frac{\left(\beta^{(1 / \alpha)-1} / \alpha\right)(\iota / \alpha)^{-N_{0}}(\pi+\gamma)}{r}+\frac{\left(e^{r t^{*}}-1\right)\left(\beta^{(1 / \alpha)-1} / \alpha\right)(\iota / \alpha)^{-N_{0}}(\pi+\gamma)}{r}\right\} \\
& =\Omega e^{-h t^{*}}+\frac{\left(\beta^{(1 / \alpha)-1} / \alpha\right)(\iota / \alpha)^{-N_{0}}(\pi+\gamma)}{r} .
\end{aligned}
$$

Obviously, inequality (44) contradicts (42), which implies (41) holds for all $t>0$. Letting $\gamma \longrightarrow 0$, we have

$$
\begin{aligned}
\lambda_{\min }(Q)\|e(t)\|^{2} \leq & V(t) \leq \Lambda(t)<\Omega e^{-h t} \\
& +\frac{\left(\beta^{(1 / \alpha)-1} / \alpha\right)(\iota / \alpha)^{-N_{0}} \pi}{-\left(\left(\ln (\iota / \alpha) / T_{a}\right)+\beta^{(1 / \alpha)}\right)} .
\end{aligned}
$$

Thus, according to (45), the error converges into

$$
\Theta=\left\{e(t) \in \mathbb{R}^{n} \mid\|e(t)\| \leq \sqrt{\frac{\left(\beta^{(1 / \alpha)-1} / \alpha\right)(\iota / \alpha)^{-N_{0}} \pi}{\lambda_{\min }(Q) \cdot(-Y)}}\right\},
$$

when $t \longrightarrow+\infty$. The proof for condition 1 is completed.

When impulsive intensity $\mu_{k} \leq-\alpha$ or $\mu_{k}>\alpha$, we have Theorem 2.

Theorem 2. When impulsive intensity $\mu_{k} \leq-\alpha$ or $\mu_{k}>\alpha$, if there exist diagonal matrices $Q>0, P>0$, scalars $\beta>0$, and $d=\operatorname{diag}\left(d_{1}, d_{2}, \ldots, d_{n}\right), \quad \bar{B}=\left(\overline{b_{i j}}\right)_{n \times n}, \quad \dot{L}=\operatorname{diag}\left(\dot{L}_{1}, \dot{L}_{2}\right.$, $\left.\ldots, \dot{L}_{n}\right), \quad \pi=N^{T} P N, \quad N=\Delta B \dot{L} \widetilde{\top}_{\max }, \quad|\Delta B|=\left(\left|\Delta b_{i j}\right|\right)_{n \times n}$, $\left.\left.\left.\widetilde{\neg}_{\max }=\operatorname{diag}(\urcorner_{\max },\right\urcorner_{\max }, \ldots,\right\urcorner_{\max }\right), \iota=\max _{k \in \mathbb{N}}\left\{\mu_{k}^{2}\right\}$, if

$$
\begin{aligned}
& \text { (1) }-2 Q d-2 Q a+Q \bar{B} \dot{L}+\dot{L} \bar{B}^{T} Q+P^{-1} Q^{2}-\beta Q<0 \\
& \text { (2) } \bar{\Upsilon}=\frac{\ln (\iota / \alpha)}{T_{a}}+\beta^{(1 / \alpha)}-\beta^{(1 / \alpha)+1}<0
\end{aligned}
$$

are satisfied, then error systems (25) achieve quasi-synchronization with error bound $\left.\sqrt{\left(\left(\beta^{(1 / \alpha)-1} / \alpha\right)(\iota / \alpha)^{N_{0}} \pi / \lambda_{\min }(Q) \cdot\right.} \cdot(-\bar{Y})\right)$ at the convergence rate $\left(h^{\prime} / 2\right)$, where $h^{\prime}$ is the unique solution of the transcendental equation $h^{\prime}+\left(\ln (\iota / \alpha) / T_{a}\right)+\beta^{(1 / \alpha)}-\beta^{(1 / \alpha)+1}=0$.

Proof. The same as the proof in Theorem 1, we obtain

$$
\begin{aligned}
e^{\beta^{(1 / \alpha)}(t-s)} \prod_{s \leq t_{k} \leq t} \frac{l}{\alpha} & \leq e^{\beta^{(1 / \alpha)}(t-s)}\left(\frac{l}{\alpha}\right)^{\left(\left(t-s / T_{a}\right)+N_{0}\right)} \\
& \leq\left(\frac{l}{\alpha}\right)^{N_{0}} e^{\left(\left(\ln (\iota / \alpha) / T_{a}\right)+\beta^{(1 / \alpha)}\right)(t-s)} .
\end{aligned}
$$

Substituting (48) into (40) yields

$$
\begin{aligned}
\Lambda(t) \leq & \left(\frac{l}{\alpha}\right)^{N_{0}} \lambda_{\max }(Q) \sum_{i=1}^{N}\left\|e_{i}(0)\right\|^{2} \frac{1}{\alpha} e^{\left(\left(\ln (\iota / \alpha) / T_{a}\right)+\beta^{(1 / \alpha)}\right) t} \\
& +\int_{0}^{t}\left(\frac{l}{\alpha}\right)^{N_{0}} e^{\left(\left(\left(\ln (\iota / \alpha) / T_{a}\right)+\beta^{(1 / \alpha)}\right)\right)(t-s)} \frac{\beta^{(1 / \alpha)-1}}{\alpha} \times(\pi+\gamma) \mathrm{d} s \\
\leq & \bar{\Omega} e^{\left(\left(\left(\ln (\iota / \alpha) / T_{a}\right)+\beta^{(1 / \alpha)}\right)\right) t}+\int_{0}^{t} \frac{\beta^{(1 / \alpha)-1}}{\alpha} e^{\left(\left(\left(\ln (\iota / \alpha) / T_{a}\right)+\beta^{(1 / \alpha)}\right)\right)(t-s)} \times\left(\frac{l}{\alpha}\right)^{N_{0}}(\pi+\gamma) \mathrm{d} s
\end{aligned}
$$


where $\bar{\Omega}=(l / \alpha)^{N_{0}}(1 / \alpha) \lambda_{\max }(Q) \sup \|e(0)\|^{2}>0$. Define $\Delta\left(h^{\prime}\right)=h^{\prime}+\left(\ln (\iota / \alpha) / T_{a}\right)+\beta^{(1 / \alpha)}-\beta^{(1 / \alpha)+1}$. Note that $\Delta(0)=\left(\ln (\iota / \alpha) / T_{a}\right)+\beta^{(1 / \alpha)}-\beta^{(1 / \alpha)+1}<0$ and $\dot{\Delta}\left(h^{\prime}\right)=1>0$. So, function $\Delta\left(h^{\prime}\right)=0$ has a unique solution $h^{\prime}>0$.

Similar to (41), we can prove the following inequality is true for all $t>0$ :

$$
\Lambda(t)<\bar{\Omega} e^{-h^{\prime} t}+\frac{\left(\beta^{(1 / \alpha)-1} / \alpha\right)(\iota / \alpha)^{N_{0}}(\pi+\gamma)}{-\left(\left(\ln (\iota / \alpha) / T_{a}\right)+\beta^{(1 / \alpha)}\right)+\beta^{(1 / \alpha)+1}} .
$$

Letting $\gamma \longrightarrow 0$, we have

$$
\begin{aligned}
\lambda_{\min }(Q)\|e(t)\|^{2} \leq & V(e(t)) \leq \Lambda(t)<\bar{\Omega} e^{-h^{\prime} t} \\
& +\frac{\left(\beta^{(1 / \alpha)-1} / \alpha\right)(\iota / \alpha)^{N_{0}} \pi}{-\left(\left(\ln (\iota / \alpha) / T_{a}\right)+\beta^{(1 / \alpha)}\right)+\beta^{(1 / \alpha)+1}} .
\end{aligned}
$$

Then, according to (51), the error converges into

$$
\bar{\Theta}=\left\{e(t) \in \mathbb{R}^{n} \mid\|e(t)\| \leq \sqrt{\frac{\left(\beta^{(1 / \alpha)-1} / \alpha\right)(\iota / \alpha)^{N_{0}} \pi}{\lambda_{\min }(Q) \cdot(-\bar{\Upsilon})}}\right\},
$$

with the convergence rate $\left(h^{\prime} / 2\right)$. So far, all proofs are over.

Remark 2. In impulsive dynamical networks or impulsive control mechanism, the impulsive effect $\mu_{k}$ is essential to the final results of the whole networks. Some previous works have discussed the impulsive effects in the synchronization of complex dynamical networks. Specifically, the impulsive effect $\mu_{k}$ is always assumed to satisfy $(-1,0)$ or $(-2,0)$. However, when a fractional-order system exchanges information with another system, the impulsive effect could either play positive roles or negative roles. In this paper, the impulsive gain $\mu_{k}$ is talked over under the value ranges: (1) $-\alpha<\mu_{k} \leq \alpha$ and $\mu_{k} \neq 0$, (2) $\mu_{k} \leq-\alpha$ or $\mu_{k}>\alpha$, where $\mu_{k}$ only have a relation with variable $\alpha$. The range of gain $\mu_{k}$ values was extended.

Remark 3. In this paper, the controller (24) contains the impulsive control part and the feedback control part. For the case $-\alpha<\mu_{k} \leq \alpha$ and $\mu_{k} \neq 0$, the impulsive effect $\mu_{k}$ plays a positive role in the quasi-synchronization. In this case, the feedback control gain $a_{i}$ could be set as zero in order to save control costs and manufacture. However, for the case $\mu_{k} \leq-\alpha$ or $\mu_{k}>\alpha$, the impulsive effect $\mu_{k}$ could hinder the synchronization of neural networks or even cause the instability of the coupled neural networks. In this case, the feedback control part could control effectively reduce the adverse effects brought by the disadvantageous impulse. In addition, if $\mu_{k}=-1$, it implies $e_{i}\left(t_{k}^{+}\right)=-e_{i}\left(t_{k}^{-}\right)$, which is impossible for impulsive control. If $\mu_{k}=0, e_{i}\left(t_{k}^{+}\right)=e_{i}\left(t_{k}\right)=0$, which means that there is no impulsive control effect.

\section{Illustrative Examples}

In this part, one example is given to show the effectiveness of our methods.

Example 1. Consider 2-dimension drive system (14) with $\alpha=0.9, f_{j}(s)=\tanh (s), j \in \mathbb{N}$. Choose $d_{1}=3.4, d_{2}=1.5$, \urcorner$_{x j}=1 L_{j}=1$,

$$
\begin{aligned}
& b_{11}\left(x_{1}\right)= \begin{cases}1.3, & \left|x_{1}\right| \leq 1, \\
1.6, & \left|x_{1}\right|>1,\end{cases} \\
& b_{12}\left(x_{2}\right)= \begin{cases}-2, & \left|x_{2}\right| \leq 1, \\
-2.45, & \left|x_{2}\right|>1,\end{cases} \\
& b_{21}\left(x_{1}\right)= \begin{cases}-0.3, & \left|x_{1}\right| \leq 1, \\
-0.6, & \left|x_{1}\right|>1,\end{cases} \\
& b_{22}\left(x_{2}\right)= \begin{cases}2.3, & \left|x_{2}\right| \leq 1, \\
2.6, & \left|x_{2}\right|>1 .\end{cases}
\end{aligned}
$$

The switching jumps and memristive connection weights of the response system (16) are the same as system (14). The initial conditions of the drive-response system are $x_{0 i}=(1.5,1)^{T}, y_{0 i}=(0.5,-0.5)^{T}$. The average impulsive interval is less than $T_{a}=0.02$ and the positive constant $N_{0}=1$.

Case 1: when $-0.9<\mu_{k} \leq 0.9$ and $\mu_{k} \neq 0$, for Theorem 1 , considering disadvantageous impulsive effects here, set $a_{i}=8(i \in N), \quad \mu_{k}=-0.7$, so we get $\iota=\mu_{k}^{2}=0.49$. Denoting $\beta=7$, then we have $\Upsilon=(\ln (\iota / \alpha)$ $\left(T_{a}\right)+\beta^{(1 / \alpha)}=-21.7099<0$. By solving conditions (4) and (5) in Theorem 1, we drive $P=\operatorname{diag}\{15.0648$, $14.9541\}, Q=\operatorname{diag}\{22.5648,21.4627\}, N=\Delta B L{\widetilde{\bar{\top}_{m a x}}}_{\max }=$ $(0.75,0.6)^{T}, \pi=\left\|N^{T} P N\right\|=13.8574$ and the corresponding error bound could be calculated as

$$
\|\|(t) \| \leq \sqrt{\frac{\left(\beta^{(1 / \alpha)-1} / \alpha\right)(\iota / \alpha)^{-N_{0}} \pi}{\lambda_{\min }(Q) \cdot(-\Upsilon)}}=0.2677 .
$$

In Figure 1, we plot the chaotic attractors of the drive-response system with initial conditions. In Figure 2, one can find that the error is under the bound 0.1752 , which is less than the error bound $\|e(t)\|$ derived from the theorem. It implies the systems achieve quasi-synchronization with a given error bound.

Case 2: when $\mu_{k}>0.9$, for Theorem 2, impulsive effect $\mu_{k}=1.3$, so $\iota=1.1^{2}=1.69$; we set the control gain $a_{i}=0, i \in \mathbb{N}$. As a similar process in Example 1, we have $\bar{\Upsilon}=\left(\ln (\iota / \alpha) / T_{a}\right)+\beta^{(1 / \alpha)}-\beta^{(1 / \alpha)+1}=-28.6372<0$ and

$$
\|\| e(t) \| \leq \sqrt{\frac{\left(\beta^{(1 / \alpha)-1} / \alpha\right)(\iota / \alpha)^{N_{0}} \pi}{\lambda_{\min }(Q) \cdot(-\bar{Y})}}=0.2356 .
$$




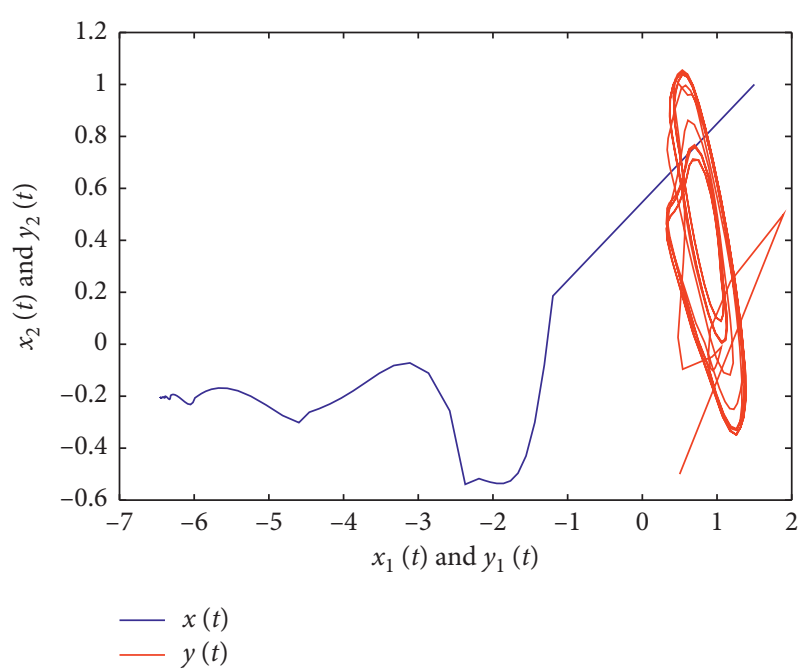

Figure 1: Chaotic attractor of the drive-response system when $\mu_{k}=-0.7$.

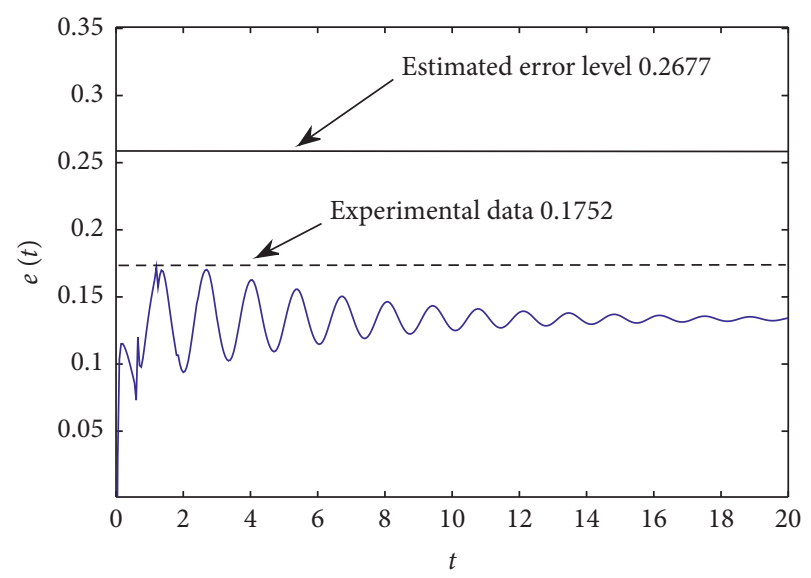

Figure 2: The synchronization error $\|e(t)\|$ when $\mu_{k}=-0.7$.

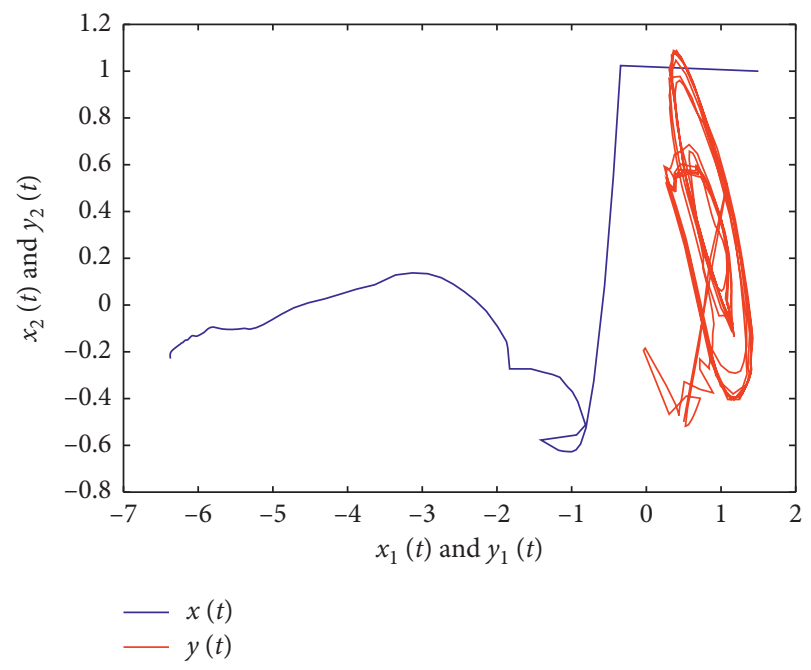

FIGURE 3: Chaotic attractor of the drive-response system when $\mu_{k}=1.3$.

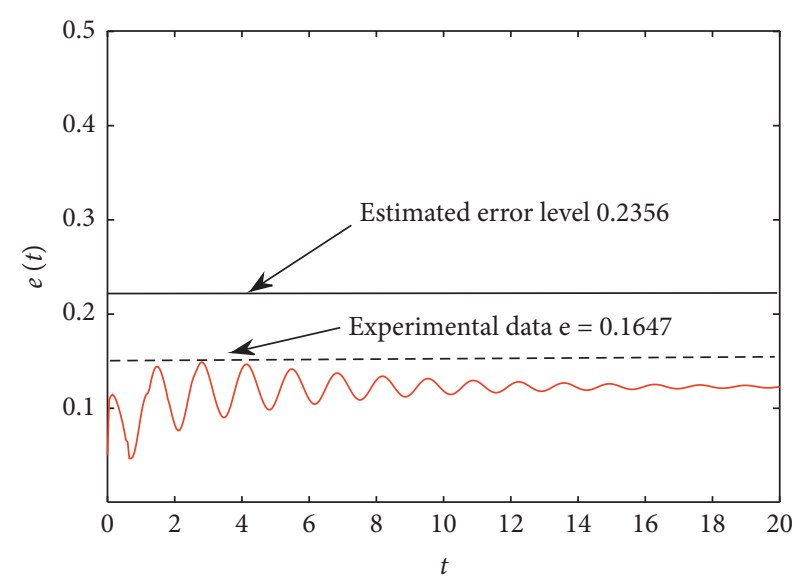

Figure 4: The synchronization error $\|e(t)\|$ when $\mu_{k}=1.3$.

The chaotic attractors of the drive-response system with initial conditions are shown in Figure 3. In Figure 4, one can find that the error is under the bound 0.1647 , which is less than the error bound $\|e(t)\|$ derived from the theorem. It can be obviously seen that the drive-response systems achieved quasi-synchronization.

\section{Conclusion}

In this paper, the problem of quasi-synchronization of delayed FMNNs is investigated. By designing an effective impulsive controller and a new fractional comparison lemma, some effective criteria for quasi-synchronization $(0<\alpha<1)$ are established. In the future, we will consider the quasi-synchronization problem of delayed FMNNs.

\section{Data Availability}

No data were used to support this study.

\section{Conflicts of Interest}

The authors declare that there are no conflicts of interest regarding the publication of this paper.

\section{Acknowledgments}

This work was supported by the Assistance Program for Future Outstanding Talents of China University of Mining and Technology (no. 2020WLJCRCZL075).

\section{References}

[1] L. Chua, "Memristor-The missing circuit element," IEEE Transactions on Circuit Theory, vol. 18, no. 5, pp. 507-519, 1971.

[2] D. B. Strukov, G. S. Snider, D. R. Stewart, and R. S. Williams, "The missing memristor found," Nature, vol. 453, no. 7191, pp. 80-83, 2008.

[3] J. M. Tour and T. He, "The fourth element," Nature, vol. 453, no. 7191, pp. 42-43, 2008.

[4] A. Wu, Z. Zeng, X. Zhu, and J. Zhang, "Exponential synchronization of memristor-based recurrent neural networks 
with time delays," Neurocomputing, vol. 74, no. 17, pp. 3043-3050, 2011.

[5] A. Wu and Z. Zeng, "Dynamic behaviors of memristor-based recurrent neural networks with time-varying delays," Neural Networks, vol. 36, pp. 1-10, 2012.

[6] A. Wu and Z. Zeng, "Lagrange stability of memristive neural networks with discrete and distributed delays," IEEE Transactions on Neural Networks and Learning Systems, vol. 25, no. 4, pp. 690-703, 2014.

[7] D. Liu, S. Zhu, and K. Sun, "Global anti-synchronization of complex-valued memristive neural networks with time delays," IEEE Transactions on Cybernetics, vol. 49, no. 5, pp. 1735-1747, 2019.

[8] L. Ye and Y. Xia, "Synchronization analysis for stochastic inertial memristor-based neural networks with linear coupling," Discrete Dynamics in Nature and Society, vol. 2020, Article ID 5430410, 14 pages, 2020.

[9] A. Wu and Z. Zeng, "Output convergence of fuzzy neurodynamic system with piecewise constant argument of generalized type and time-varying input," IEEE Transactions on Systems, Man, and Cybernetics: Systems, vol. 46, no. 12, pp. 1689-1702, 2016.

[10] S. P. Adhikari, C. Changju Yang, H. Hyongsuk Kim, and L. O. Chua, "Memristor bridge synapse-based neural network and its learning," IEEE Transactions on Neural Networks and Learning Systems, vol. 23, no. 9, pp. 1426-1435, 2012.

[11] S. Lawrence, C. L. Giles, A. C. Ah Chung Tsoi, and A. D. Back, "Face recognition: a convolutional neural-network approach," IEEE Transactions on Neural Networks, vol. 8, no. 1, pp. 98-113, 1997.

[12] J. Schmidhuber, "Deep learning in neural networks: an overview," Neural Networks, vol. 61, pp. 85-117, 2015.

[13] I. Podlubny, Fractional Differential Equations, Academic Press, Cambridge, MA, USA, 1999.

[14] X. Li and Y. Ge, "New results on synchronization of fractional-order memristor-based neural networks via state feedback control," Discrete Dynamics in Nature and Society, vol. 2020, Article ID 2470972, 11 pages, 2020.

[15] J. Yu, C. Hu, and H. J. Jiang, “-Stability and-synchronization for fractional-order neural networks," Neural Networks, vol. 35, no. 35, pp. 82-87, 2012.

[16] G. Velmurugan, R. Rakkiyappan, and J. Cao, "Finite-time synchronization of fractional-order memristor-based neural networks with time delays," Neural Networks, vol. 73, pp. 36-46, 2016.

[17] J. Chen, Z. Zeng, and P. Jiang, "Global Mittag-Leffler stability and synchronization of memristor-based fractional-order neural networks," Neural Networks, vol. 51, pp. 1-8, 2014.

[18] L. Chen, R. Wu, J. Cao, and J.-B. Liu, "Stability and synchronization of memristor-based fractional-order delayed neural networks," Neural Networks, vol. 71, pp. 37-44, 2015.

[19] A. Wu and Z. Zeng, "Global mittag-leffler stabilization of fractional-order memristive neural networks," IEEE Transactions on Neural Networks and Learning Systems, vol. 28, no. 1, pp. 206-217, 2017.

[20] C. Chen and Z. Ding, "Projective synchronization of nonidentical fractional-order memristive neural networks," Discrete Dynamics in Nature and Society, vol. 2019, Article ID 8743482, 11 pages, 2019.

[21] W. He, F. Qian, J. Lam, G. Chen, Q.-L. Han, and J. Kurths, "Quasi-synchronization of heterogeneous dynamic networks via distributed impulsive control: error estimation, optimization and design," Automatica, vol. 62, pp. 249-262, 2015.
[22] S. Ding, Z. Wang, and H. Zhang, "Quasi-synchronization of delayed memristive neural networks via region-partitioningdependent intermittent control," IEEE Transactions on Cybernetics, vol. 49, no. 12, pp. 4066-4077, 2018.

[23] H. Rao, Y. Xu, H. Peng, R. Lu, and C.-Y. Su, "Quasi-synchronization of time delay markovian jump neural networks with impulsive-driven transmission and fading channels," IEEE Transactions on Cybernetics, vol. 50, no. 9, p. 4121, 2020.

[24] Z. Tang, J. H. Park, Y. Wang, and J. Feng, "Distributed Impulsive Quasi-Synchronization of Lur'e Networks With Proportional Delay," IEEE Transactions on Cybernetics, vol. 49, no. 8, pp. 3105-3115, 2019.

[25] W. He, F. Qian, Q.-L. Han, and J. Cao, "Lag quasi-synchronization of coupled delayed systems with parameter mismatch," IEEE Transactions on Circuits and Systems I: Regular Papers, vol. 58, no. 6, pp. 1345-1357, 2011.

[26] Z. Tang, J. H. Park, and J. Feng, "Impulsive effects on quasisynchronization of neural networks with parameter mismatches and time-varying delay," IEEE Transactions on Neural Networks and Learning Systems, vol. 29, no. 4, pp. 908-919, 2018.

[27] X. Liu, T. Chen, J. Cao, and W. Lu, "Dissipativity and quasisynchronization for neural networks with discontinuous activations and parameter mismatches," Neural Networks, vol. 24, no. 10, pp. 1013-1021, 2011.

[28] X. Huang, Y. Fan, J. Jia, Z. Wang, and Y. Li, "Quasi-synchronisation of fractional-order memristor-based neural networks with parameter mismatches," IET Control Theory \& Applications, vol. 11, no. 14, pp. 2317-2327, 2017.

[29] L. Zhang and Y. Yang, "Different impulsive effects on synchronization of fractional-order memristive BAM neural networks," Nonlinear Dynamics, vol. 93, no. 2, pp. 233-250, 2018.

[30] A. Abdurahman, H. Jiang, and Z. Teng, "Finite-time synchronization for memristor-based neural networks with timevarying delays," Neural Networks, vol. 69, pp. 20-28, 2015. 